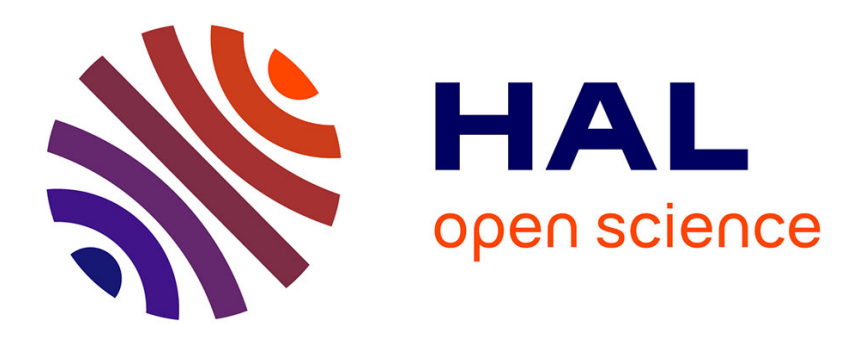

\title{
Transit time during the interparticle percolation process
}

Franck Lominé, Luc Oger

\section{To cite this version:}

Franck Lominé, Luc Oger. Transit time during the interparticle percolation process. Physical Review E: Statistical, Nonlinear, and Soft Matter Physics, 2010, 82 (4), pp.041301. 10.1103/PhysRevE.82.041301 . hal-00531644

\section{HAL Id: hal-00531644 \\ https://hal.science/hal-00531644}

Submitted on 21 Jul 2016

HAL is a multi-disciplinary open access archive for the deposit and dissemination of scientific research documents, whether they are published or not. The documents may come from teaching and research institutions in France or abroad, or from public or private research centers.
L'archive ouverte pluridisciplinaire $\mathbf{H A L}$, est destinée au dépôt et à la diffusion de documents scientifiques de niveau recherche, publiés ou non, émanant des établissements d'enseignement et de recherche français ou étrangers, des laboratoires publics ou privés. 


\title{
Transit time during the interparticle percolation process
}

\author{
Franck Lominé* \\ Research Institute of Civil Engineering and Mechanics, UMR CNRS 6183, University of Nantes, \\ 58 rue Michel Ange, BP 420-44606, Saint-Nazaire, Cedex, France \\ Luc Oger ${ }^{\dagger}$ \\ Institut de Physique de Rennes, UMR CNRS-UR1 6251, Université de Rennes 1, \\ 263 Avenue du Général Leclerc-35042 Rennes Cedex, France
}

(Received 10 January 2010; revised manuscript received 24 August 2010; published 6 October 2010)

\begin{abstract}
A numerical investigation of jamming effect during the spontaneous interparticle percolation process of small beads through an unconsolidated porous media has been performed. The size ratio between the moving beads and the ones building up the porous medium was chosen larger than the geometrical trapping threshold: $\xi_{c}=\left(\frac{2}{\sqrt{3}}-1\right)^{-1}=6.464 \ldots$. In this paper, we used the discrete element method algorithm to study the rebounds of particles on the top of the porous medium and the transit times of an assembly of particles through it. Several parameters such as the number of injected particles, the size ratio between beads, and the energy restitution coefficient are investigated. This study leads to give some important results of the evolution of the transit time versus the contiguous volume occupied by the percolating particles.
\end{abstract}

DOI: 10.1103/PhysRevE.82.041301 PACS number(s): 45.70. - n, 83.80.Fg, 81.05.Rm, 02.70.Ns

\section{INTRODUCTION}

The segregation, which can occur during the handling of a granular material, is an important problem. Due to external forces, a granular assembly can be subjected to grain reorganizations. Then, inhomogeneous spatial distribution of grains can be observed due to differences in grains properties. In this paper, we focus our attention on grains with different sizes. The well known example of size segregation is the "Brazil Nuts problem" [1]. Our work, presented in this paper, is related to size segregation of grains under the gravity effect. For this study, we observe the interparticle percolation process of particles through a packing of larger spheres. Previous experiments [2,3] shown that collective effects can lead to behavior differences compared to cases when only one particle travels inside the packing of larger spheres [4-7]. These previous experiments and numerical simulations put in evidence that these collective effects are mainly governed by the size of the grain species and by the number of percolating grains. In other words, these collective effects, on the transit time [2] and on the dispersion of particles [3], result essentially in jamming effects during the flow of the small particles through the porous structure of larger spheres. We are agreeing with the new improved definition of jamming made by [8] as the jammed states is defined as stable equilibrium states, where small load increments cause only small displacements, and large unrestricted particle motions will not occur. First, we present our numerical program, based on discrete element method, to perform the study of the spontaneous interparticle percolation phenomenon. In a second part, results are presented and analyzed by considering separately the penetration of the particle blob in the porous medium, and its flow through the entire packing of larger spheres.

\footnotetext{
*franck.lomine@univ-nantes.fr

†luc.oger@univ-rennes1.fr
}

\section{NUMERICAL SETUP}

In this section, we present the set-up that we developed and used to study the flow of a group of particles through a packing made with static larger spheres. It is directly inspired from the experimental set-up used by Lomine and Oger [3]. The algorithm that we chose and developed is based on Discrete Element Method of soft spheres [known as molecular dynamics (MD)] and it runs as follow. The static part of the simulation (i.e., the two sphere packings) is created with a numerical gravity like deposition which is performed with the Powell's algorithm [3,9]. A small packing of spheres, with diameter $d$, is generated and placed above another packing of larger spheres $(D)$ which constitute the porous medium. The distance which separates the two packings is set to one diameter of larger spheres. Both packings are stable and isotropic. The Powell's algorithm can let us adjust the porosity to a desired value which was set to 0.4 in order to be consistent with the experimental set-up previously used [3]. To avoid limited size effects, the porous medium is considered as infinite in transverse directions. This is realized by using a periodic construction algorithm.

The dynamic part of the simulation is performed with a molecular dynamics of soft sphere model [10]. During collisions, particles are allowed to slightly overlap each other. The value $\delta$ of this overlap permits to calculate interaction forces, for a pair of colliding particles, through the use of a force model. In this work we use the widely sprayed "linear spring dashpot" model $[3,11,12]$ to calculate normal force and the Cundall and Strack model $[3,10]$ for the tangential force. Time integration of particle positions is achieved with an implementation of the Verlet algorithm [13]. For efficiency, collision detection is made with the use of cells and a doubly linked-list [3] and all parts of the algorithm are parallelized using MPI. More details, about our simulation program can be found in Ref. [3]. To illustrate this model, a snapshot, taken from a simulation of flow of 20000 particles, is presented in Fig. 1. 


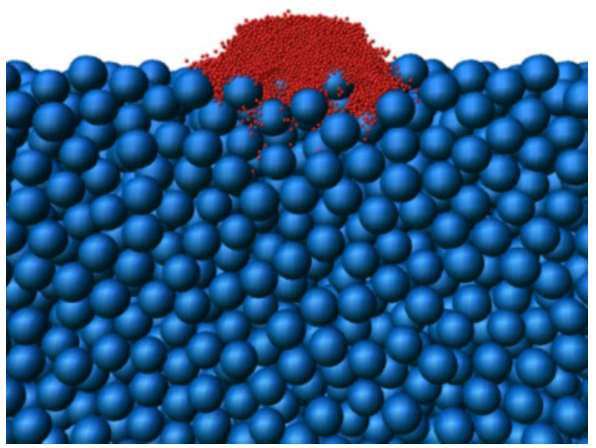

FIG. 1. (Color online) Snapshot of flow of 20000 small particles through a porous medium. Note that, for convenience, only half system is represented.

Our numerical model has been validated with confrontation with numerous experimental results $[2,3,14]$. The use of numerical simulation in conjunction with our previous experimental studies let us observe interparticle percolation phenomenon and the jamming effect during such flow through porous material. We present now, in the next section, the results of this study.

\section{RESULTS}

First when particles are launched on top of the porous medium, a quantity of them rebounces on the upper packing surface. Indeed according to the presence of a pore or not under the small particles, the time needed by a particle to enter in the porous medium can vary. Moreover, due to the presence of other particles, some particles cannot enter in the porous space since pores are already filled with other particles. These effects can lead to a penetration delay that has been experimentally and numerically observed [3].

Second, during the crossing of the first grain layers of the packing of larger spheres, we observe a transitional regime with velocity thermalizations (defined as granular temperature freezing). This regime was put in evidence by Lomine and Oger [3]. Indeed, in dilute granular flow, the fluctuation of grains velocities is quantified by the measure of so-called granular temperature: higher the temperature is, higher the velocities of the grains are, and by opposition the decrease of the velocities can be modeled as a freezing.

It is interesting to point out that two very different behaviors for the moving particles (fast horizontal displacement when the packing fraction is small in one hand, slow global displacement when the packing fraction is high) can generate very similar effects: a slow vertical falling velocity. In other words, they lead to similar results: a loss of kinetic energy.

Overall, we can observe two distinct phases during the interparticle percolation phenomenon. The first one is how particles penetrate in the medium, and the second one is how they flow in it. Moreover it is essential to ask which coupling can appear between them?

Numerically, it is possible to avoid taking into account these external rebounds of the moving particles, before entering in the porous medium, during the flow property determination (like the mean transit time for example). This leads

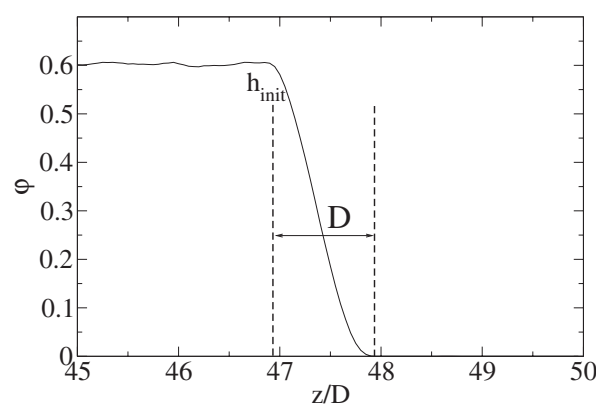

FIG. 2. Packing fraction profile of the packing of spheres of diameter $D$. The height $h_{\text {init }}$, which permits to define $t_{\text {init }}$ is also represented.

to the ability to separate the influence of the rebound phase on global flow properties, what is almost impossible experimentally.

The following section presents our numerical results and is divided as follow: first, we present and analyze results about the jamming, which occur during the entrance of particles in the porous medium, then we study its influence on the flow of particles in the underneath grain layers. All these effects will be analyzed in term of their influence on transit times.

\section{A. Jamming during entrance of particles in the porous medium}

In this part we are looking for how particles enter in the porous structure. First, we can see that the porous medium is perfectly homogeneous along the vertical axis almost up to the last layer of large particles (Fig. 2). The packing fraction fluctuation is only observed in a $D$ thickness which permit us to define the entrance zone as this order of length. We define $t_{\text {init }}$ as the time for a particle to pass through the first larger grain layer at height $h_{\text {init }}$ from the top of the porous medium (Fig. 2). We can obtain this time for every particle. For example, Fig. 3 shows probability distribution function of times $t_{\text {init }}$ for a launch of different numbers of particles with $N=500, N=5000$ and $N=8000$ for $D / d=16$.

It illustrates clearly the fact that, when the number of particles injected in the porous medium increases, the tail of the probability distribution function of times $t_{\text {init }}$ is larger. In other words, the more particles are injected in the porous

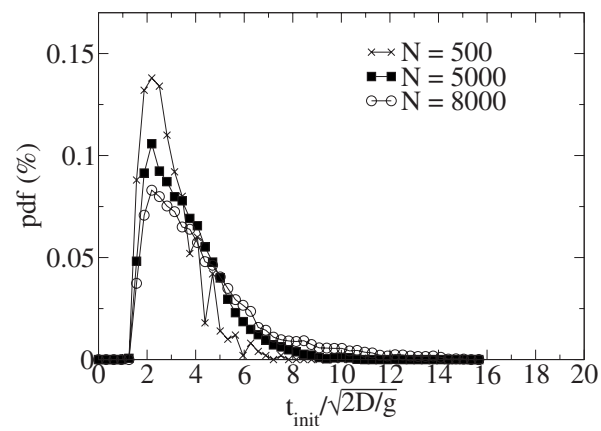

FIG. 3. Distributions of times $t_{\text {init }}$ for launches of different numbers of particles with $N=500, N=5000$ and $N=8000$ for $D / d=16$. 
medium, the more they have difficulties to pass the first layer of grains. This can be easily understood as a consequence of a jamming effect due to the limited pore volume immediately accessible for the flowing particles under their falling zone (also visible in Fig. 1). Moreover, we can already notice that the more probable value of $t_{\text {init }} / \sqrt{2 D / g}$ is close to a value of 2 with a decreasing probability when the amount of particle increases. In such case, the mean value of $t_{\text {init }} / \sqrt{2 D / g}$ increases. The normalization term is linked to the classical free fall over one large bead height $(1 / \sqrt{2 D / g})$. So, this more probable value corresponds to the free fall time over a distance of $2 D$, integrating the distance between the two initial packing position and the first grain thickness. Let's look at each effect separately. Indeed, this jamming effect during entrance is made of two contributions, as already mentioned: rebounds of particles on the top surface of the porous medium and their penetration in the porous medium.

Several parameters can influence the way particles penetrate in the packing of larger spheres. First, we made a series of numerical simulations to determine how the mean time $\left\langle t_{\text {init }}\right\rangle$ is influenced by the volume of falling particles. This volume depends on two parameters that can be independently set in our simulations: the number $N$ of percolating particles and the size ratio $D / d$.

Instead of considering the number of particles $N$ or the size ratio $D / d$, it can be convenient to cast in term of injected volume or in a better way in term of filled pores. That is why, we have defined a dimensionless value $N_{p}$ [2], which can link the number of falling particles and the large pore size,

$$
N_{p}\left(N, \frac{d}{D}\right)=\frac{N \frac{4}{3} \pi\left(\frac{d}{2}\right)^{3}}{\Phi_{\mathrm{RCP}} \frac{D^{3}}{12}(\sqrt{2}-2 \Omega)} \approx 31.5 N\left(\frac{d}{D}\right)^{3},
$$

where $\Phi_{\mathrm{RCP}}=0.64$ is the random close packing limit and $\Omega$ $=3 \arccos (1 / 3)-\pi$ is the solid angle of the tetrahedron composed by four spheres of diameter $D$. The interstitial volume of this tetrahedron corresponds to the minimal accessible one for the percolating particles of diameter $d$; in practice the size distribution of these volumes starts at this value and can reach a size up to the volume of the sphere $D$, but the shape of this distribution is always similar for all disordered packings [15]. The upper part of Eq. (1) represents the volume of small particles injected in the porous structure and the lower part is an estimation of the accessible volume of the smallest pore of the porous medium. The parameter $N_{p}$ is then useful to analyze phenomenon in term of number of contiguous filled pores [3]. We can notice that it is possible to use this defined parameter $N_{p}$ when both $N$ and $d / D$ vary. In the following simulations, only one parameter influence will be studied at a time. To clarify situations between varying $N$ and varying $d / D$, we use the notation $N_{p}\left(N, \frac{d_{0}}{D_{0}}\right)=N_{p}^{N}$ when dealing with $N_{p}$ at a fixed size ratio $d_{0} / D_{0}$. In a same manner, the notation $N_{p}\left(N_{0}, \frac{d}{D}\right)=N_{p}^{d / D}$ will be adopted with fixed number of particles $N_{0}$ and varying $d / D$.

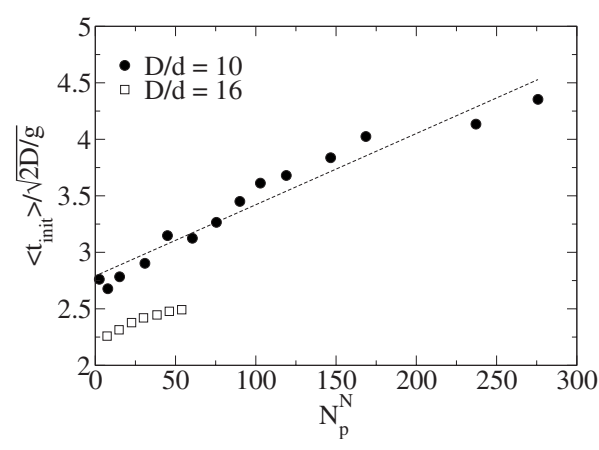

FIG. 4. Evolution of mean time $\left\langle t_{\text {init }}\right\rangle$ for different number of particles. This evolution is given in terms of number of filled pores via the parameter $N_{p}^{N}$. A linear adjustment is also represented.

\section{Effect of the number of particles}

Figure 4 shows dependency of the dimensionless mean time $\left\langle\widetilde{t}_{\text {init }}\right\rangle=\left\langle t_{\text {init }}\right\rangle / \sqrt{2 D / g}$ with $N_{p}^{N}$ for several simulations and for two values of size ratio $D / d$. Figure 4 illustrates that $\left\langle\tilde{t}_{\text {init }}\right\rangle$ increases with $N_{p}^{N}$, i.e.: with the number of particles falling down at the same time. For small values of $N_{p}^{N},\left\langle t_{\text {init }}\right\rangle$ is close to the value of the time for falling over a distance of $2 D$. As the number of injected particles increases, $\left\langle t_{\text {init }}\right\rangle$ almost increases linearly with $N_{p}^{N}$. We can also observed in Fig. 4 that these values increase when the size ratio $D / d$ decreases. A series of simulations with various ratio $D / d$ have been performed and results are analyzed in the following section through the parameter $N_{p}^{d / D}$.

\section{Influence of the size ratio $D / d$}

To characterize more precisely the dependency of the dimensionless $\left\langle\tilde{t}_{\text {init }}\right\rangle$ on $D / d$ previously observed in Fig. 4, let us consider Fig. 5 which presents results obtained for various values of size ratio expressed in termed of volume with the parameter $N_{p}^{d / D}$. Figure 5 shows that dimensionless $\left\langle\tilde{t}_{\text {init }}\right\rangle$ increases proportionally with $N_{p}^{d / D}$. Indeed, for small $N_{p}^{d / D}$ (i.e., for small $d / D$ ), particles can more easily pass through the first grain layer. Moreover as observed previously, Fig. 5 confirms that the dimensionless $\left\langle t_{\text {init }}\right\rangle$ is more important when the number of particles $(N)$ is higher.

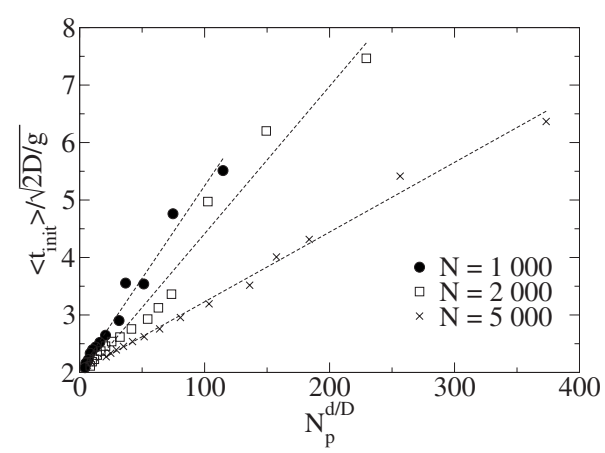

FIG. 5. Dependency of mean $t_{\text {init }}$ with $N_{p}^{d / D}$ for $N=1000, N$ $=2000$ and $N=5000$. 


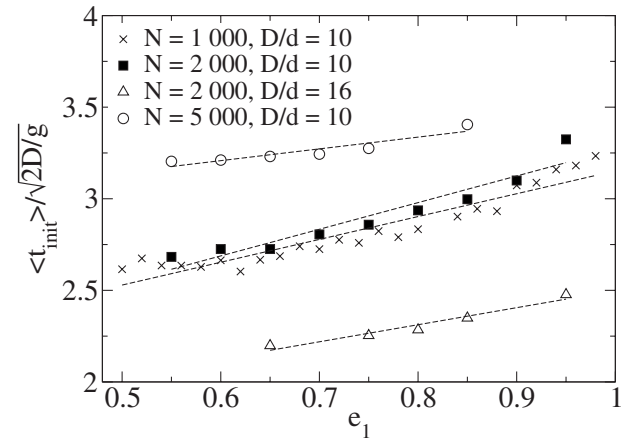

\section{Effect of energy dissipation}

During spontaneous interparticle percolation phenomenon, the particles moved due to collisions which facilitate more or less the appearance of jamming effects during the entrance phase. A physical parameter that can control their ability to stay in dense region or to move to less dense region is the energy restitution coefficient of a collision. Dispersion of particles due to collisions during the flow was investigated in previous work [3]. Here we are only interested in the effect which occurs between the launch of particles and their initial crossing down to the height $h_{\text {init }}$. Due to the fact that we only consider here the first large grain layer, collisions that can affect the mean time $\left\langle t_{\text {init }}\right\rangle$ are the collisions of particles on the upper porous medium surface. Here, two restitution coefficients can be defined. The first one is the restitution coefficient which characterizes a collision between a small moving bead and a large immobile bead. The second one is the restitution coefficient that characterizes a collision between two percolating moving particles. These two restitution coefficients will be respectively denoted $e_{1}$ and $e_{2}$ in the rest of this paper. In order to analyze influence of energy restitution on jamming effects during fall of particles, a series of simulations with various $e_{1}$ and $e_{2}$ has been carried out.

Dependency of $\left\langle\widetilde{t}_{\text {init }}\right\rangle$ on $e_{1}$ and $e_{2}$ are presented respectively in Fig. 6(a) and Fig. 6(b). Figure 6(a) shows that the mean time needed to penetrate in the porous medium increases with $e_{1}$. This time increases when energy dissipation decreases. This result is due to higher rebounds of particles on the porous medium surface with less energy dissipation. The particles need more time to dissipate their kinematics energy in order to enter in pore holes. As several particles try to enter at the same time in the porous structure through the same pore, an increase of the number of falling particles leads to a penetration time delay as we have observed previously. This can also be observed in Fig. 6(a).

Figure 6(b) shows that the mean time $\left\langle\tilde{t}_{\text {init }}\right\rangle$ does not truly depend on the restitution coefficient $e_{2}$. So energy dissipation during entrance of the particle blob in the porous structure is mainly due to rebounds of particle on the porous medium surface made with larger spheres. This observation confirms that the freezing of the granular temperature at the entrance of the porous medium observed in other experimental analysis does not contribute to slow down the initial transit time. But this behavior can play an important role for the rest of the flow as the falling particles would have less energy for continuing to fall.
All results presented here show that the time needed to enter in the porous medium can vary according to the many parameters that govern experiments of interparticle percolation of small particles through a packing of larger spheres. Indeed, we have seen that the mean time for crossing the first grain layer depends on the number of particles, the size ratio and the energy restitution coefficients.

\section{B. Effect of jamming on percolation process}

After these considerations, we are going to analyze the effect of these parameters on the mean transit time of the blob of particles through the entire packing of height $H$. To bypass the entrance phase jamming phenomenon, we will use a definition of transit time that does not take into account the time needed to penetrate in the porous space. We call $t_{\text {init }}^{i}$ the time for a particle $i$ to cross the first layer and $t^{i}$ the time required to reach the output of the porous media. The mean transit time, with previous remark, is then given by

$$
\left\langle t^{\star}\right\rangle=\frac{\sum_{i=1}^{N}\left(t^{i}-t_{\text {init }}^{i}\right)}{N} .
$$

We can notice that such calculation cannot be done experimentally with the experimental set-up presented in Ref. [3].

\section{Effect of the number of particles}

Jamming in interparticle percolation phenomenon depends on the number of particles falling down. Lomine and Oger [2] have experimentally shown existence of three flow regimes according to the number of particles falling down

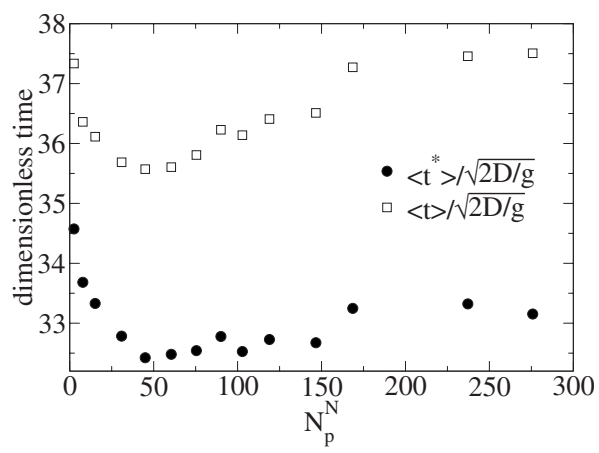

FIG. 7. Evolution of dimensionless times: $\langle t\rangle$ (global) and $\left\langle t^{\star}\right\rangle$ (corrected) with $N_{p}^{N}$ with fixed value of $D / d=10$. 

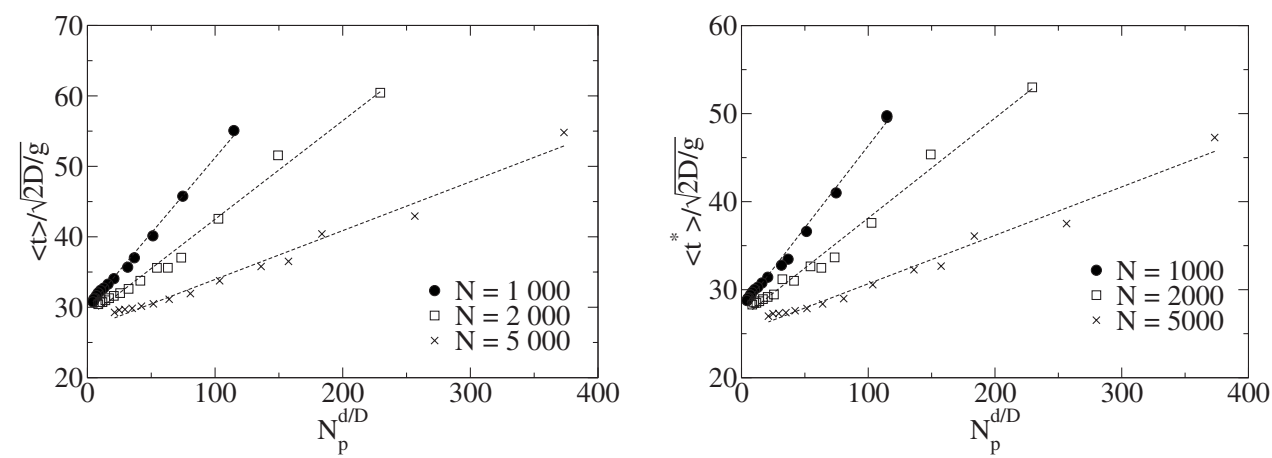

FIG. 8. Evolutions of mean times $\langle t\rangle$ (a) and $\left\langle t^{\star}\right\rangle$ (b) with $N_{p}^{d / D}$ for $N=1000, N=2000$ and $N$ $=5000$. Linear adjustments in dashed line are also represented. through the porous media. In the first one, they observed that the mean transit time decreases when the number of falling particles grows. As $N_{p}^{N}$ increases, the particles loose more and more energy by collisions with their very close neighbors. Then, the particles are mainly falling down directly in the lower pore structure, without bouncing around. As the number of small beads increases further, an equilibrium between the direct falling process and the interparticle collision events is obtained. In this steady gravity regime, the mean transit time is quite independent of $N_{p}^{N}$. If we continue to increase the number of falling beads, they observed a third regime in which the particle velocities slow down due to the crossing of a large number of particles simultaneously through the same pore. In fact, this flow regime, where jamming occurs, can be divided into two subregimes. First, the dependency of the slowdown on $N_{p}^{N}$ is important. This behavior reflects a beginning of a jamming effect of pores located in the flow zone as noted previously. Then, the jamming effect grows with $N_{p}^{N}$ and the increase of the mean transit time with $N_{p}^{N}$ is less important due to accumulation of particles in connecting pores. In this study, we have determined the mean time transit $\left\langle t^{\star}\right\rangle$ for a series of simulations with different number of particles. The results obtained are presented in Fig. 7. This shows that our simulation model is able to reproduce the different flow regimes observed experimentally. Moreover these flow regimes can also be observed even if we make abstraction of the time needed by the particle blob to penetrate the porous structure.

\section{Effect of the size ratio $\mathrm{D} / \mathrm{d}$}

Figure 8 presents the dependency of the dimensionless mean transit time on $N_{p}^{d / D}$ for three numbers $N$ of particles. Figure 8(a) reveals a linear relation between the mean transit time of the particle blob and $N_{p}^{d / D}$. The Fig. 8(b) illustrates the dependency of $\left\langle t^{\star}\right\rangle$ on $N_{p}^{d / D}$. By making comparison between the Figs. 8(a) and 8(b), we can deduce that the mean transit time of the particle blob to flow inside the porous medium is proportional to $N_{p}^{d / D}$ even without the entrance phase effect. Indeed, as seen previously in Fig. $5,\left\langle t_{\text {init }}\right\rangle$ increases proportionally with $N_{p}^{d / D}$. These linear dependencies with $N_{p}^{d / D}$ confirm the linear dependency with $N_{p}^{d / D}$ of the global mean transit time observed in Fig. 8(a)

\section{Effect of energy dissipation}

We have seen in Sec. III A that the mean time $\left\langle t_{\text {init }}\right\rangle$ mainly depends on the restitution coefficient $e_{1}$, which characterized a collision between a moving particle and a sphere of the porous medium. Figures 9 (a) and 9(b) presents, respectively, evolution of the velocities $H /\langle t\rangle$ and $H /\left\langle t^{\star}\right\rangle$ with $\left(1-e_{1}\right)^{1 / 4}$. In both graphs, we observed a linear relation. The theoretical study of Wilkinson and Edwards [16], based on the analyze of Brownian motion for the case of a single particle flow, reported the same relation between the mean velocity of the flow and $\left(1-e_{1}\right)^{1 / 4}$. Results presented in Figs. 9(a) and 9(b) confirm this fact also when several particles flow at the same time in the porous structure. We have neglected to draw the same curves with $e_{2}$ as no dependency is observed.

\section{CONCLUSION}

In this paper, we have investigated jamming effects which can occur when a packing of small particles flows through a porous medium made of larger spheres. Jamming effects can first arise during the penetration of particles into the porous medium, and second during their flow inside it. We have also
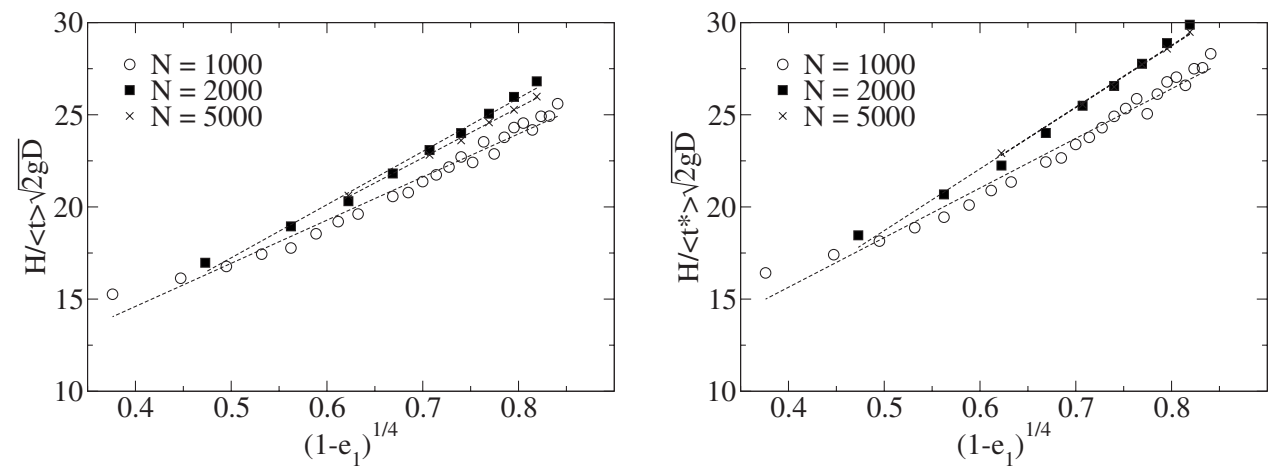

FIG. 9. Dimensionless velocities of a blob of particles with the restitution coefficient $e_{1}$ for $D / d$ $=10$ and for $N=1000\left(N_{p}^{N} \approx 31\right)$, $N=2000\left(N_{p}^{N} \approx 60\right)$ and $N=5000$ $\left(N_{p}^{N} \approx 146\right)$. Linear adjustments are also represented. 
shown that the mean transit time $\left\langle t_{\text {init }}\right\rangle$ of particles to cross the first grain layer increases linearly with the parameter $N_{p}$ $\left(N_{p}^{N}\right.$ and $\left.N_{p}^{d / D}\right)$. This analysis of the influence of the number of particles demonstrates the existence of jamming effect during the penetration of the particle blob, and the presence of collective effects during the flow inside the porous space. These last ones can also result in jamming effect leading to a nondependency on $N_{p}$ of the mean transit time, for a large number of particles. As observed by Lomine and Oger [2], in such flow regime, some pores are fully occupied by smallest particles and no flow toward the pores of the underneath grain layer can take place until these ones are vacated. In this work, an analysis of the influence of size ratio $D / d$ revealed that the mean transit time $\langle t\rangle$ and the mean time $\left\langle t_{\text {init }}\right\rangle$ are proportional to the ratio $(d / D)^{3}$. These evolutions are due to jamming effect coming from the volume occupied by the small particles. An increase of the ratio between the volume of small particle blob and the pore volume lead to an increase of the penetration time as well as an increase of the time required to percolate through the packing of large spheres. Energy dissipation influence on transit time has also been investigated. It has been pointed out that the restitution coefficient of collisions, between the small particles and the larger spheres of the porous medium, is the only one which has influence on the particles penetration inside the porous medium. When the energy dissipation due to rebounds of particles on the surface of the porous medium decreases, particles needs more time to enter in the porous structure. Moreover, this study shows that the mean transit time of a blob of particles evolves linearly with $\left(1-e_{1}\right)^{1 / 4}$.
[1] A. Rosato, K. J. Strandburg, F. Prinz, and R. H. Swendsen, Phys. Rev. Lett. 58, 1038 (1987).

[2] F. Lominé and L. Oger, J. Stat. Mech.: Theory Exp. (2006) P07019.

[3] F. Lominé and L. Oger, Phys. Rev. E 79, 051307 (2009).

[4] J. Bridgwater and N. D. Ingram, Trans. Inst. Chem. Eng. 49, 163 (1971).

[5] J. Masliyah and J. Bridgwater, Trans. Inst. Chem. Eng. 52, 31 (1974).

[6] J. Bridgwater, Powder Technol. 15, 215 (1976).

[7] I. Ippolito, L. Samson, and J. P. Hulin, Eur. Phys. J. E 3, 227 (2000).

[8] K. Bagi, Granular Matter 9, 109 (2007).

[9] M. J. Powell, Powder Technol. 25, 45 (1980).
[10] P. A. Cundall and O. D. L. Strack, Geotechnique 29, 47 (1979).

[11] L. Oger, S. Savage, D. Corriveau, and M. Sayed, Mech. Mater. 27, 189 (1998).

[12] L. E. Silbert, D. Ertas, G. S. Grest, T. C. Halsey, D. Levine, and S. J. Plimpton, Phys. Rev. E 64, 051302 (2001).

[13] L. Verlet, Phys. Rev. 159, 98 (1967).

[14] L. Oger and F. Lominé, in Powders and Grains, edited by R. Garcia-Rojo, H. J. Herrmann, and S. McNamara (Taylor \& Francis Group, New York, 2005), Vol. 1, pp. 57-61.

[15] H. J. Frost and R. Raj, J. Am. Ceram. Soc. 65, C19 (1982).

[16] D. R. Wilkinson and S. F. Edwards, Proc. R. Soc. London, Ser. A 381, 33 (1982). 